\title{
Mechanical support of cardinal/uterosacral ligaments improves $O A B$ symptoms: implications for management
}

\author{
Peter Petros ${ }^{1,2}$ (D) \\ Received: 12 April 2018 / Accepted: 24 May 2018 / Published online: 20 June 2018 \\ (C) The International Urogynecological Association 2018
}

\section{Dear Editors.}

The interesting, statistically validated study by Zacharakis et al. demonstrated relief of overactive bladder (OAB) by mechanical support of the vaginal apex [1]. The authors demonstrated that OAB symptom occurrence was independent of the degree of prolapse. They stated that the pessary test "may have both a therapeutic aspect and/or an estimative role before surgical reconstruction" and "The causal association between these two entities is also supported by the observation that treating patients for POP (surgery, pessaries) may result in an improvement in OAB symptoms." Interventions such as those described, which mechanically support a ligament, are known as simulated operations. The best known simulated operation is control of urine loss during coughing by unilateral upward pressure at midurethra with a hemostat to support the pubourethral ligament. Another is manual replacement and support of a prolapse to unmask latent stress incontinence. A third is the gentle insertion of the lower half of a bivalve speculum to support cardinal/uterosacral ligaments (CL/ USL) during clinical examination to relieve urge and pelvic pain, as described by Wu et al. in 2013 [2].

The authors refer to surgical cure of OAB following pelvic organ prolapse (POP) reconstruction. This statement was validated in a recent study by Liedl et al. in a group of 70-year-old women $(n=611)$ [3] with second- to fourth-degree pelvic organ prolapse (POP). They achieved a 90\% cure for prolapse, $85 \%$ for urge incontinence $(n=310), 83 \%$ for frequency $(n=317)$,

Peter Petros

pp@kvinno.com

1 University of NSW Professorial Department of Surgery, St Vincent's Hospital Sydney, Sydney, NSW, Australia

2 University of Western Australia School of Mechanical and Chemical Engineering, Perth, WA, Australia and $68 \%$ for nocturia $(n=254)$ by shortening and reinforcing CL/USL with a tensioned sling.

The explanation put forward by the authors to explain both the surgical cure mentioned in the text and the effect of the pessary was "the distension of the bladder wall, which may trigger the stretch receptors of the urothelium resulting in detrusor contractions." This is very similar to the statement of the 1990 Integral Theory[AQ: This statement requires a ref. and citation pls], which stated that symptoms of urgency, frequency, and nocturia were mainly due to a prematurely activated, but otherwise normal, micturition reflex.

\section{Compliance with ethical standards}

Conflict of interest None.

\section{References}

1. Zacharakis D, Grigoriadis T, Pitsouni E, Kypriotis K, Vogiatzis N, Athanasiou S. Assessment of overactive bladder symptoms among women with successful pessary placemen. Int Urogynecol J. 2018;29:571-7. https://doi.org/10.1007/s00192-017-3461-x.

2. Wu Q, Luo L, Peter Petros P. Mechanical support of the posterior fornix relieved urgency and suburethral tenderness. Pelviperineology. 2013;32:55-6.

3. Liedl B, Inoue $\mathrm{H}$, Sekiguchi $\mathrm{Y}$, et al. Is overactive bladder in the female surgically curable by ligament repair? Cen Eur J Urol. 2017;70:51-7. 